\title{
Active Structural Error Suppression in MEMS Vibratory Rate Integrating Gyroscopes
}

\author{
Chris C. Painter, Student Member, IEEE, and Andrei M. Shkel, Associate Member, IEEE
}

\begin{abstract}
Due to restrictive tolerancing in microfabrication, structural imperfections that reduce performance of fabricated micro devices are typical. In microelectromechanical vibratory gyroscopes, feedback control is a common strategy in attempting to correct the imperfections. However, a purely feedback control can be insufficient for compensation of all the errors, requiring post processing in the form of laser trimming to achieve higher levels of performance. In this paper, we explore another alternative: the design and implementation of a dual stage control architecture with self-calibration and feedback capabilities. The self-calibrative portion of the control identifies and electronically "trims" large imperfections, while the feedback control compensates for remaining small nonidealities and in-operation perturbations. Presented here is an algorithm for in-situ imperfection identification based on the dynamic response of the device. A realization of the dual stage control architecture is proposed for a gyroscope using nonlinear electrostatic parallel plate actuators. Modeling and simulation results which demonstrate successful compensation of imperfections with the proposed architecture for a device with $10 \%$ fabrication error appearing in the form of stiffness nonidealities and subjected to further $1 \%$ in-run perturbations are presented.
\end{abstract}

Index Terms-Error suppression, microelectromechanical systems (MEMS), rate integrating gyroscopes, smart MEMS.

\section{INTRODUCTION}

A S MICROELECTROMECHANICAL systems (MEMS) inertial sensors have begun to proliferate more into rate and tactical grade application markets [1], the current demand is for inertial sensors with higher precision and long term performance. In its current maturity, fabrication technologies fall below the tolerancing required for these demands, requiring additional error suppression capabilities to achieve design goals. Currently, in order to operate with the highest precision, vibratory gyroscopes typically include active feedback control to compensate for fabrication imperfections [2]-[4]. However, as it will be illustrated in this paper, when imperfections are large compared to the measured Coriolis force, compensation cannot be achieved with a purely feedback control without interfering with the Coriolis measurements. These interferences cause scale factor and drift errors in the gyroscope, resulting in degraded performance. In these cases, both post processing such as laser

Manuscript received October 30, 2002; revised May 21, 2003. This work was supported by the Department of Defense under a 2001 National Defense Science and Engineering Graduate Fellowship. The associate editor coordinating the review of this paper and approving it for publication was Dr. Lina Sarro.

The authors are with the Department of Mechanical and Aerospace Engineering, University of California, Irvine, CA 92612 USA (e-mail: cpainter@ uci.edu; ashkel@uci.edu).

Digital Object Identifier 10.1109/JSEN.2003.817165 trimming [5], ion beam milling [6], or selective material deposition [7] and feedback control are required.

The demand for improved performance has also given rise to a new paradigm of "smart" devices [9] with enhanced capabilities, such as active structural compensation, self-calibration, and signal processing integrated on the same chip. Under this new paradigm, we present an alternative to potentially costly and time consuming post processing of each individual device by implementation of a dual stage architecture (Fig. 1) that utilizes both self-calibrative capabilities for elimination of large structural imperfections and feedback control for elimination of smaller in-run perturbations. A gyroscope using this architecture would be fabricated with the self-calibration capabilities built onto the same chip to enable in situ system identification, such as stiffness and damping parameters. From the results of the self-calibration, the device would be capable of electronically "trimming" the large imperfections in the form a feedforward control with gains based off the anisoelasticity parameters. The feedforward control would work in tandem with a feedback control which would compensate for small perturbations arising during the normal operation of the device.

A prototype rate integrating gyroscope [8] (Fig. 2) is used as an illustrative model to demonstrate the dual stage architecture principles. Section II of the paper describes the fundamental dynamical principles of single mass vibratory rate integrating gyroscopes. In Section III, we present the effect of anisoelasticity on gyroscope performance and the design of a purely feedback type of control architecture. We show that when a device with large structural imperfections uses a purely feedback control, this degrades the device performance. Section IV details the calibration algorithm used to identify anisoelasticity and Section $\mathrm{V}$ describes the feedforward control implementation realized using electrostatic parallel plates. Concluding remarks are in Section VI.

\section{GYROSCOPE DYNAMICS}

A single mass vibratory rate integrating gyroscope can be modeled as a lumped mass-spring system operating in its first two fundamental in-plane modes [Fig. 3(a)]. The lumped massspring dynamics of an ideal system are expressed in the rotating coordinate frame $(\mathrm{X}, \mathrm{Y})$ by [10]

$$
\begin{aligned}
& \ddot{x}+\omega_{n}^{2} x-2 \Omega \dot{y}=0 \\
& \ddot{y}+\omega_{n}^{2} y+2 \Omega \dot{x}=0
\end{aligned}
$$

where $x$ and $y$ are deflections along the $\mathrm{X}$ and $\mathrm{Y}$ axis, respectively, $\omega_{n}$ is the natural frequency, and $\Omega$ is the input angular 


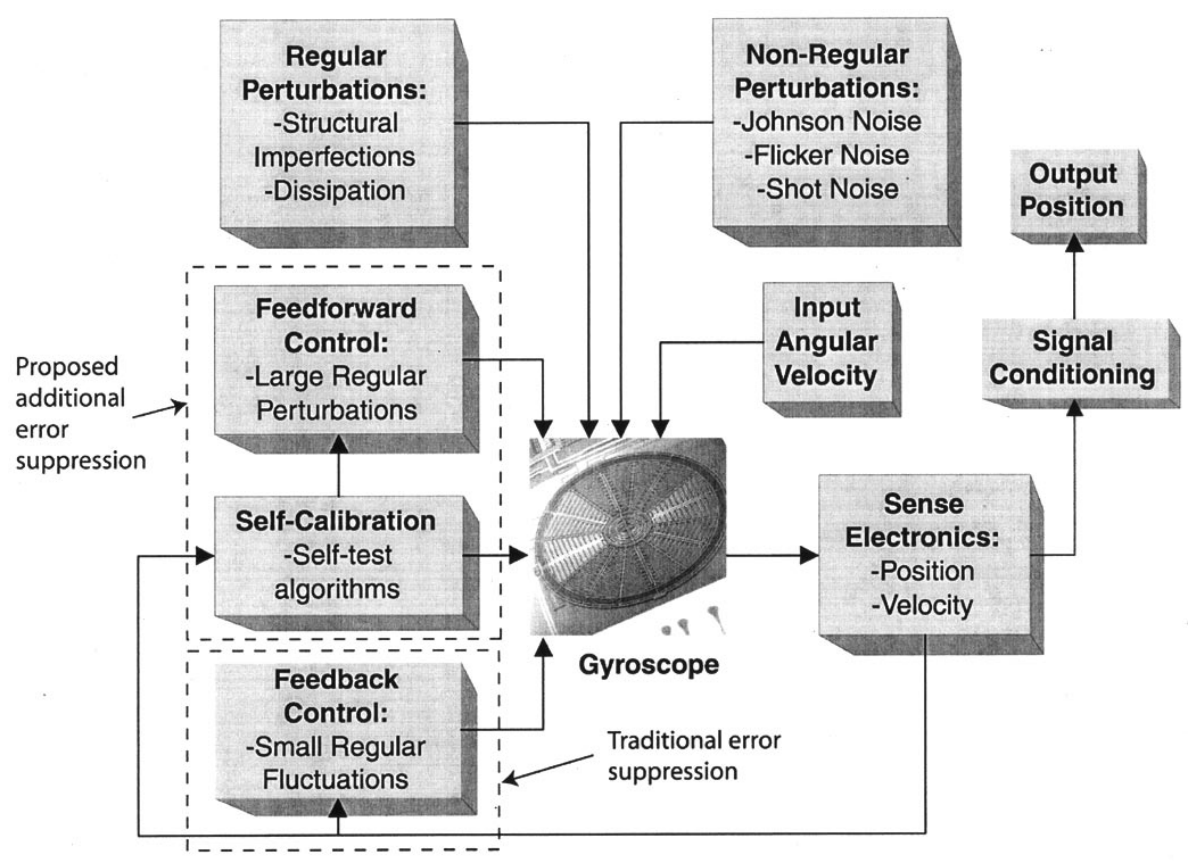

Fig. 1. Smart MEMS gyroscope would include self-calibration capabilities built on-chip for detection and compensation of deterministic perturbations.

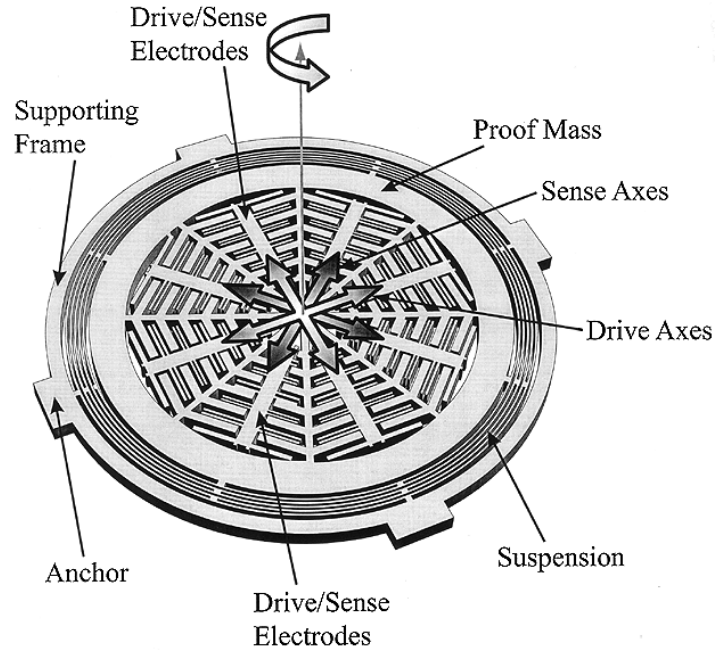

Fig. 2. Studied rate integrating gyroscope [8] consists of a freely vibrating proof mass attached to a concentric six-ring suspension. Stationary electrodes interwoven throughout the mass sustain motion and allow measurement of the Coriolis-induced precession angle. The precession angle is proportional to the angle of rotation of the device.

velocity. In a nonrotating system $(\Omega=0)$, the solution is an ellipse of semi-major axis length $a$, semi-minor axis length $b$, and oriented at an angle $\phi$ from the X-Y axes [Fig. 3(b)]. A convenient way of expressing the trajectory of the system is using these elliptical "orbital" variables [11] $(a, b$, and $\phi)$ which are common in orbital and celestial mechanics. If the ellipse is oriented with the $\mathrm{X}-\mathrm{Y}$ axes, the solution can be expressed by

$$
\begin{aligned}
& x=a \cos \left(\omega_{n} t+\gamma_{0}\right) \\
& y=b \sin \left(\omega_{n} t+\gamma_{0}\right)
\end{aligned}
$$

where $\gamma_{0}$ defines the initial "orbital angle" $\gamma$, designating the starting point of the mass on the ellipse. Angle $\phi$ accounts for the orientation of the ellipse, giving the complete generalized elliptical equations as

$$
\begin{aligned}
& x=a \cos \phi \cos \theta-b \sin \phi \sin \theta \\
& y=a \sin \phi \cos \theta+b \cos \phi \sin \theta \\
& \dot{x}=-\omega_{n} a \cos \phi \sin \theta-\omega_{n} b \sin \phi \cos \theta \\
& \dot{y}=-\omega_{n} a \sin \phi \sin \theta+\omega_{n} b \cos \phi \cos \theta
\end{aligned}
$$

where

$$
\theta=\omega_{n} t+\gamma_{0} .
$$

Based off the position and velocity, the orientation angle can be instantly calculated by

$$
\tan 2 \phi=\frac{2\left(\omega_{n}^{2} x y+\dot{x} \dot{y}\right)}{\omega_{n}^{2}\left(x^{2}-y^{2}\right)+\left(\dot{x}^{2}-\dot{y}^{2}\right)} .
$$

While $x$ and $y$ are changing very quickly over one period of oscillation ( 10,000 vibrations per second), the orbital parameters $a, b$, and $\phi$ remain nearly constant over one period. Thus, averaging techniques may be implemented to approximate the long term behavior of the slowly varying orbital parameters. This technique is useful in evaluating the effect of perturbations on the gyroscopic system [12]. In the interest of space, a terse background is presented, a more general explanation of this technique can be found in [13] and in the scope of gyroscopic systems in [11] and [14]. The initial dynamic system in (1) can be presented in state form as

$$
\dot{u}=A u+f(u)
$$

where $u=\{x, y, \dot{x}, \dot{y}\}$ and $f(u)$ are small perturbations such as the Coriolis force, anisoelasticity, and damping. The homogeneous solution when $f(u)=0$ is

$$
u=g(z, t)
$$




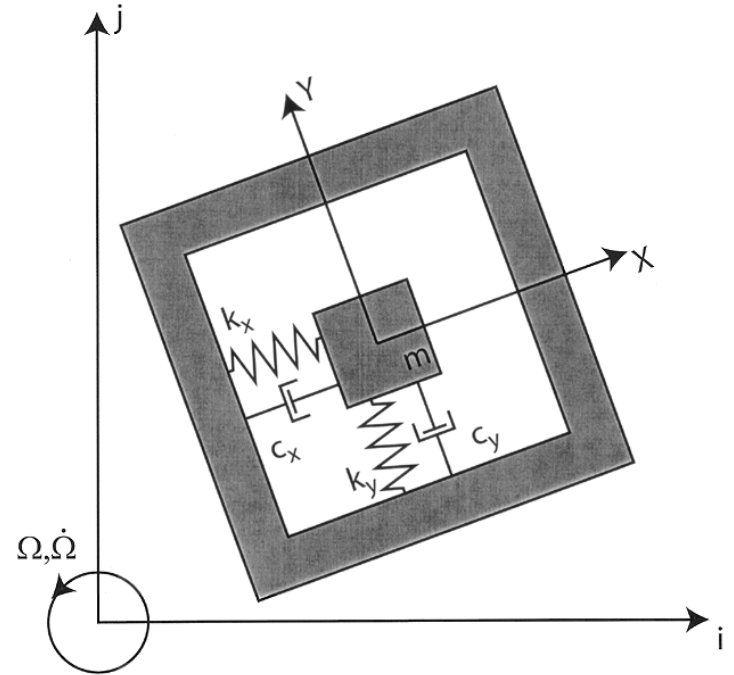

(a)

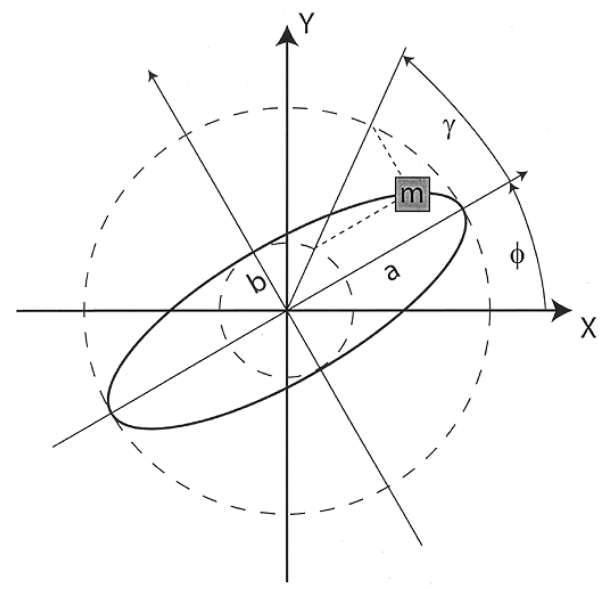

(b)

Fig. 3. (a) Gyroscope is modeled as a two-degree-of-freedom lumpec mass-spring system. $\{i, j, k\}$ is the inertial coordinate system and $\{X, Y, Z\}$ is the coordinate system attached to the rotating device. (b) In the absence of rotation, the mass trajectory is an ellipse with semi-major axis length $a$, semi-minor axis $b$, inclination angle $\phi$, and orbital angle $\gamma$.

where $z=\left\{a, b, \phi, \gamma_{0}\right\}$ is an array of initial condition determined constants. To solve for the particular solution, we use variation of parameters, where $z=z(t)$. Differentiating (6) gives

$$
\begin{aligned}
& \dot{u}=\dot{g}+J \dot{z} \\
& J=\frac{\partial g}{\partial z} .
\end{aligned}
$$

Using the fact that $\dot{g}=A g$ and substituting back into (5) gives

$$
\begin{aligned}
& \dot{z}=J^{-1} h(z, t) \\
& h=f(g(z, t)) .
\end{aligned}
$$

Time averaging over one period of oscillation $T$ yields

$$
\begin{aligned}
& \dot{z} \approx \frac{1}{T} \int_{0}^{T} J^{-1} h(z, t) d t \\
& T=\frac{2 \pi}{\omega_{n}} .
\end{aligned}
$$

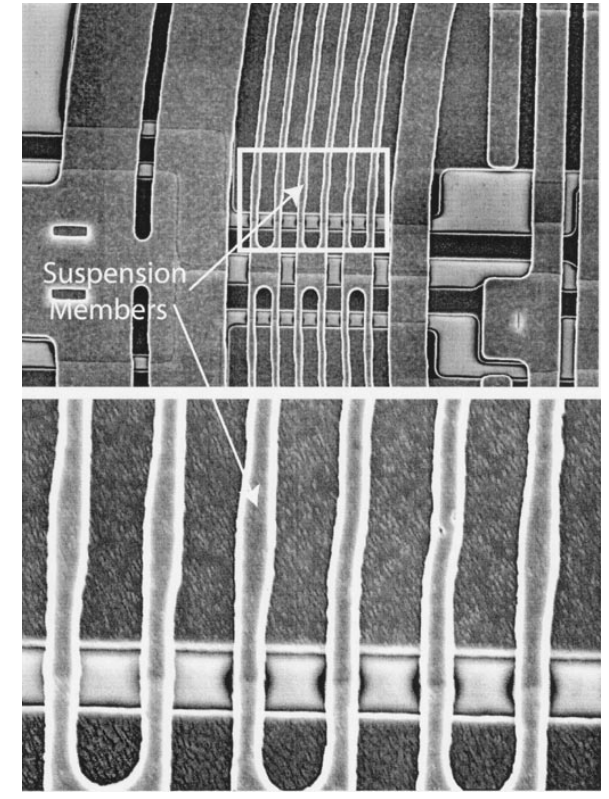

Fig. 4. Anisoelasticities are an unavoidable byproduct of fabrication due to tolerancing restrictions of optical lithography, deposition, and chemical etching. In the gyroscope, one manifestation of this is nonconsistent geometry in the concentric ring suspension members.

If the only perturbations are the Coriolis force, then this process leads to the orbital state equations given as [11]

$$
\begin{aligned}
& \dot{a}=0 \\
& \dot{b}=0 \\
& \dot{\phi}=-\Omega \\
& \dot{\gamma}=\omega_{n} .
\end{aligned}
$$

Thus, we see that when the device is rotated at a constant velocity $\Omega$, the vibration pattern will precess with the same angular velocity, but in the opposite direction with respect to a viewer in the moving frame. Assuming the oscillation pattern initially coincides with the X-Y coordinate system $(\phi=0)$, the inclination of the ellipse $\phi$, which can be found at any given time by (4), is exactly equal to the negative angle of rotation of the device

$$
\phi=-\int_{0}^{t} \Omega d t .
$$

A device operating on these principles mechanically integrates any input angular rate and an output angular displacement can be resolved without integrating any electronic signals. An important property is that even if $\Omega$ varies in time, (8) is still valid. Since (8) is twice differentiable, it can be observed that the angular acceleration $\dot{\Omega}$ of the vibration pattern precession is equal to the negative angular acceleration of the device. Thus, the calculated precession angle is invariant to changes in the angular rate. This important property has also been observed in vibrating shells [15].

\section{ANISOELASTICITY}

Anisoelasticities are an unavoidable byproduct of fabrication due to tolerancing restrictions of optical lithography, deposition, and chemical etching (Fig. 4). Anisoelasticities disrupt symmetry of the suspension, causing frequency mismatch and mode 
coupling. Assuming negligible nonlinear effects, the equations of motion with these stiffness nonidealities become

$$
\begin{aligned}
& m \ddot{x}+k_{x x} x+k_{x y} y-2 m \Omega \dot{y}=0 \\
& m \ddot{y}+k_{y y} y+k_{y x} x+2 m \Omega \dot{x}=0
\end{aligned}
$$

where $m$ is the lumped mass approximation for the gyroscope and $k_{x x}, k_{y y}, k_{x y}$, and $k_{y x}$ are the nonideal stiffness terms (due to energy conservation, $k_{x y}=k_{y x}$ ). By the properties of matrices, the stiffness terms can be expressed as the sum of a diagonal, rotation, and skew-symmetric matrix

$$
\begin{aligned}
& {\left[\begin{array}{ll}
k_{x x} & k_{x y} \\
k_{y x} & k_{y y}
\end{array}\right]} \\
& \quad=\left[\begin{array}{cc}
\xi_{1} & 0 \\
0 & \xi_{1}
\end{array}\right]+\left[\begin{array}{cc}
\xi_{2} & \xi_{3} \\
\xi_{3} & -\xi_{2}
\end{array}\right]+\left[\begin{array}{cc}
0 & \xi_{4} \\
-\xi_{4} & 0
\end{array}\right] .
\end{aligned}
$$

Here, if the stiffness matrix is symmetric, $\xi_{4}$ is naturally zero. Another convenient way of expressing anisoelasticity is in terms of the principal stiffness values $K_{1}$ and $K_{2}$ and the angular mismatch angle $\alpha$ of the principal axes of elasticity with the $\mathrm{X}-\mathrm{Y}$ coordinate system (Fig. 5), where $\mathrm{X}$ and $\mathrm{Y}$ correspond to the intended reference frame of the gyroscope (defined by the layout of the device). In these terms, the stiffness nonidealities are written as [12]

$$
\begin{aligned}
& \xi_{1}=k_{n} \\
& \xi_{2}=h \cos (2 \alpha) \\
& \xi_{3}=h \sin (2 \alpha)
\end{aligned}
$$

where $k_{n}$ is average of the principal axes stiffness values, $2 k_{n}=$ $\left(K_{1}+K_{2}\right)$, and $h$ is the mismatch between the principal axes stiffness values, $2 h=\left(K_{1}-K_{2}\right)$. In the presence of small anisoelasticities, the long term effect on the elliptical state equations can be approximated by [11]

$$
\begin{aligned}
\dot{a} & =\frac{-b}{2 \omega_{n}}\left(\xi_{4}-\xi_{2} \sin 2 \phi+\xi_{3} \cos 2 \phi\right) \\
\dot{b} & =\frac{a}{2 \omega_{n}}\left(-\xi_{4}-\xi_{2} \sin 2 \phi+\xi_{3} \cos 2 \phi\right) \\
\dot{\phi} & =\frac{a b}{\omega_{n}\left(a^{2}-b^{2}\right)}\left(\xi_{2} \cos 2 \phi+\xi_{3} \sin 2 \phi\right)-\Omega \\
\dot{\gamma} & =\frac{1}{2 \omega_{n}}\left(-\xi_{1}-\xi_{2} \cos 2 \phi-\xi_{3} \sin 2 \phi\right)+\omega_{n} .
\end{aligned}
$$

We see that anisoelasticity interferes with the precession angle $\phi$ while the device is driven with ellipticity $(b \neq 0)$. However, by running the device with as small ellipticity as possible $(b=0)$, the effects of anisoelasticity on the precession will be eliminated. Based on this fact, the ideal gyroscope should be run with as close to a linear oscillation pattern as possible [Fig. 6(a)]. Further, by choosing a control effort with the same topology as the skew symmetric matrix $\left(\xi_{4} \neq 0\right)$, the ellipticity can be driven to zero without interfering with the precession pattern. This is the basis of a feedback control system for structural error suppression. An appropriate feedback control which will compensate

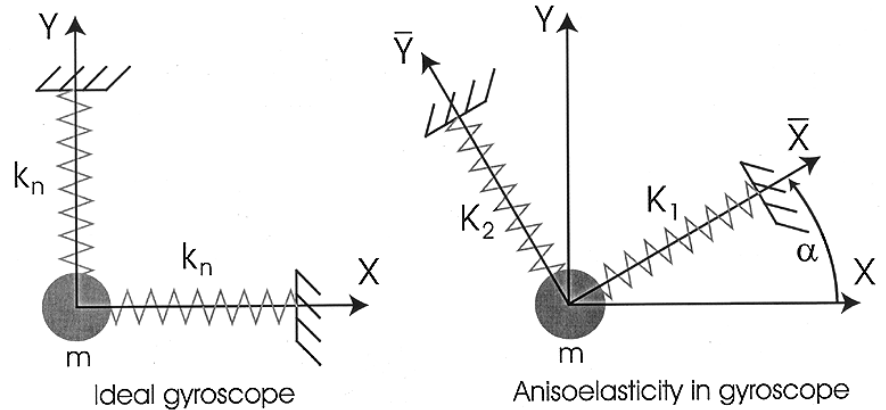

Fig. 5. In the ideal gyroscope, the principal axes of elasticity have equal stiffness $\left(k_{n}\right)$ and coincide with the $\mathrm{X}-\mathrm{Y}$ coordinate system, where $\mathrm{X}$ and $\mathrm{Y}$ correspond to the intended reference frame of the gyroscope (defined by the layout of the device). In the presence of imperfections, there is a mismatch in the principal stiffness values $2 h=\left(K_{1}-K_{2}\right)$ and an angular mismatch of the principal axes from the X-Y coordinate system by an angle $\alpha$.

for anisoelasticity while not interfering with the precession is of the form [10]

$$
\left\{\begin{array}{l}
F_{x} \\
F_{y}
\end{array}\right\}=-\gamma_{1} \cdot P \cdot S^{T} \cdot\left\{\begin{array}{l}
x \\
y
\end{array}\right\}
$$

where $\gamma_{1}$ is a constant gain, $\mathrm{S}$ is a unity gain $2 \times 2$ skew symmetric matrix, and $\mathrm{P}$ is angular momentum defined as

$$
P=(x \dot{y}-y \dot{x}) .
$$

The underlying assumptions for this control is that anisoelasticity is sufficiently small [e.g., $\alpha=10^{\circ}$ and $h$ corresponds to $1 \%$ of the ideal stiffness, Fig. 6(b)]. For larger errors [e.g., $\alpha=10^{\circ}$ and $h$ corresponds to $5 \%$ of the ideal stiffness, Fig. 6(c)], the controller, while compensating for the errors, also interferes with the measured precession angle (demonstrated in Section V). One fabricated prototype gyroscope (Fig. 7) shows much larger errors ( $\alpha=45$ and h corresponds to $72 \%$ of the ideal stiffness) and while this is an extreme example and can vary from run to run and device to device, it illustrates that some level of structural imperfections will always be present. Thus, it is necessary to "trim" the anisoelasticites to a level where a feedback control can provide compensation. Besides post processing, this can be accomplished electronically through the use of a feedforward type control architecture where the control gains are set based on the anisoelasticity parameters.

\section{IDENTIFICATION OF ERRORS}

The first step in implementing the system for electronic "trimming" of the imperfections is to develop an algorithm used to determine the structural imperfections as part of a self-calibration diagnostic test. Previous work on system identification has demonstrated a systematic approach to identifying anisoelasticity of a torsional "rocking" MEMS rate gyroscope using model synthesis and Markov parameters [16]. However, in linear mass gyroscopes, there are certain characteristics of dynamic motion that allow for a simpler algorithm for identifying anisoelasticities. Here, we present this algorithm 


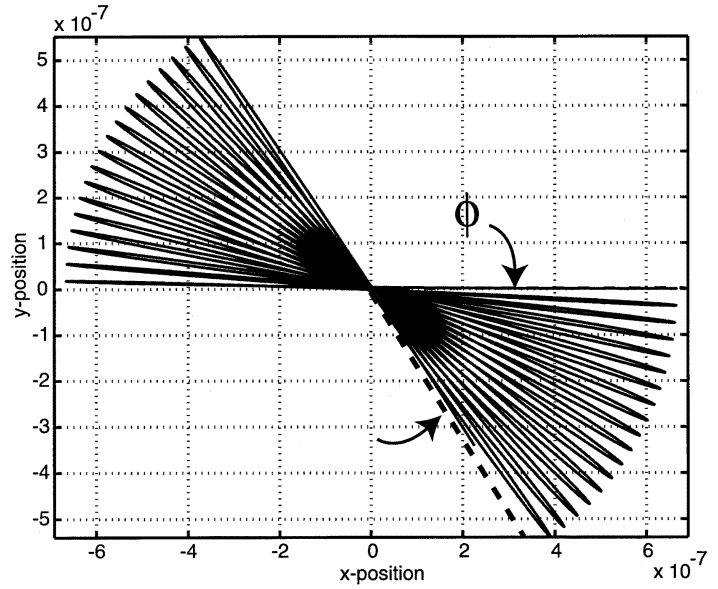

(a)

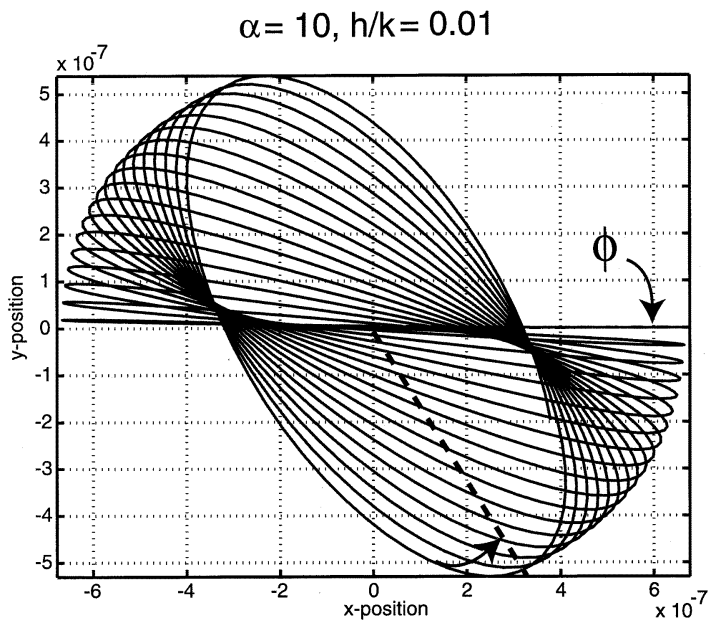

(b)

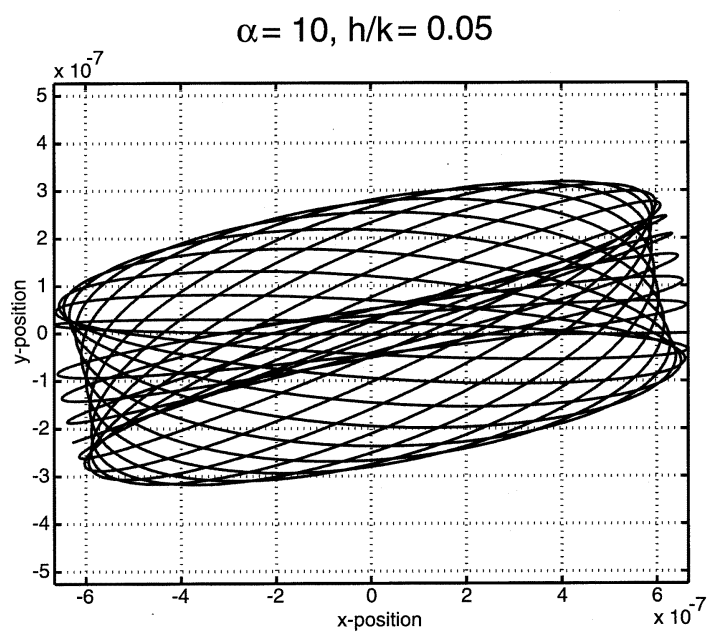

(c)

Fig. 6. (a) In the presence of an input angular rotation, the line of oscillation of an ideal gyroscope would be observed to precess by an angle $\phi$ with respect to the rotating coordinate system. (b) With small anisoelasticities, the line of oscillation is disrupted as it precesses. These anisoelasticities enter into the equations for the measured precession angle, causing degradation of performance. (c) Large anisoelasticities are even more disruptive, completely eliminating precession.

developed for identifying errors based off the dynamic response of the device.

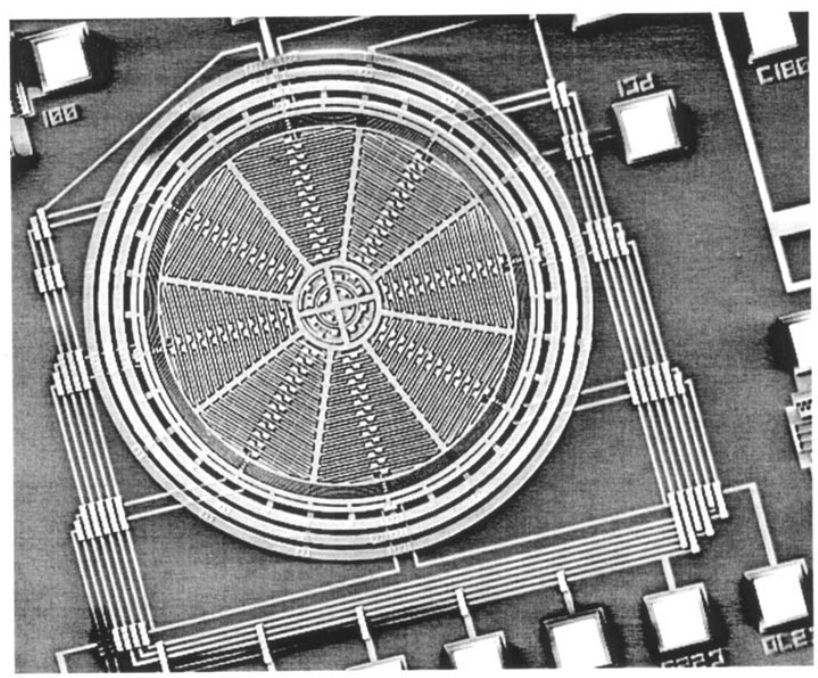

(a)

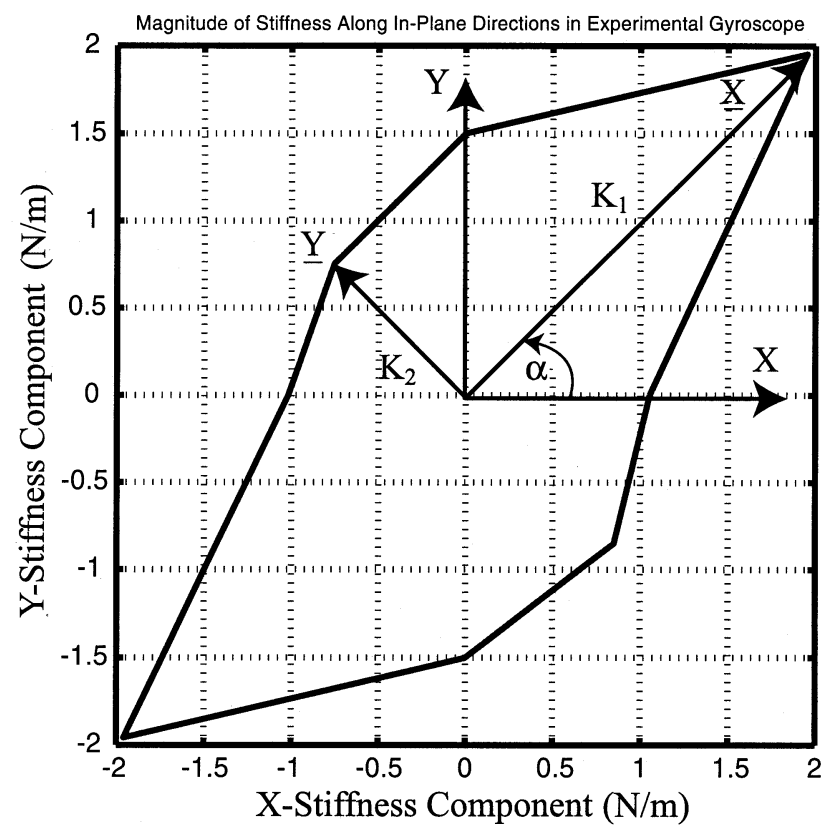

(b)

Fig. 7. (a) Prototype surface machined rate integrating gyroscope was fabricated using JDS Uniphase's MUMPS process. (b) In the prototype gyroscope, the stiffness along the four axes is estimated by observing the natural frequency along each direction and knowing the ideal mass of the system. The plot of stiffness magnitude along the four directions shows large mismatches between the principal axis stiffnesses $K_{1}$ and $K_{2}\left(K_{1}-K_{2} / K_{1}+K_{2}=72 \%\right)$ and coupling (rotation of the stiffness axes by angle $\alpha=45$ ).

\section{A. Algorithm}

Substituting (10) and (11) into (9) and solving for $x$ and $y$ assuming no angular rotation $(\Omega=0)$, no damping, and an initial deflection of $x_{0}$ and $y_{0}$, yields

$$
\begin{aligned}
& \left\{\begin{array}{l}
x \\
y
\end{array}\right\} \\
= & {\left[\begin{array}{cc}
1+\cos 2 \alpha & \sin 2 \alpha \\
\sin 2 \alpha & 1-\cos 2 \alpha
\end{array}\right]\left\{\begin{array}{l}
x_{0} \\
y_{0}
\end{array}\right\} \cos \sqrt{\left(\omega_{n}^{2}+\frac{h}{m}\right)} t } \\
& +\left[\begin{array}{cc}
1-\cos 2 \alpha & -\sin 2 \alpha \\
-\sin 2 \alpha & 1+\cos 2 \alpha
\end{array}\right]\left\{\begin{array}{l}
x_{0} \\
y_{0}
\end{array}\right\} \cos \sqrt{\left(\omega_{n}^{2}-\frac{h}{m}\right)} t .
\end{aligned}
$$




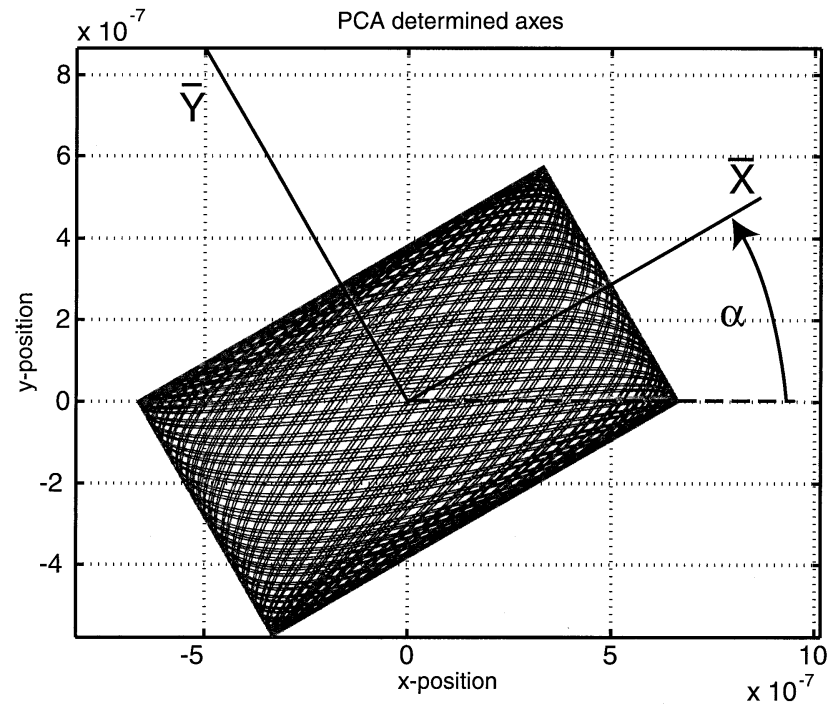

(a)

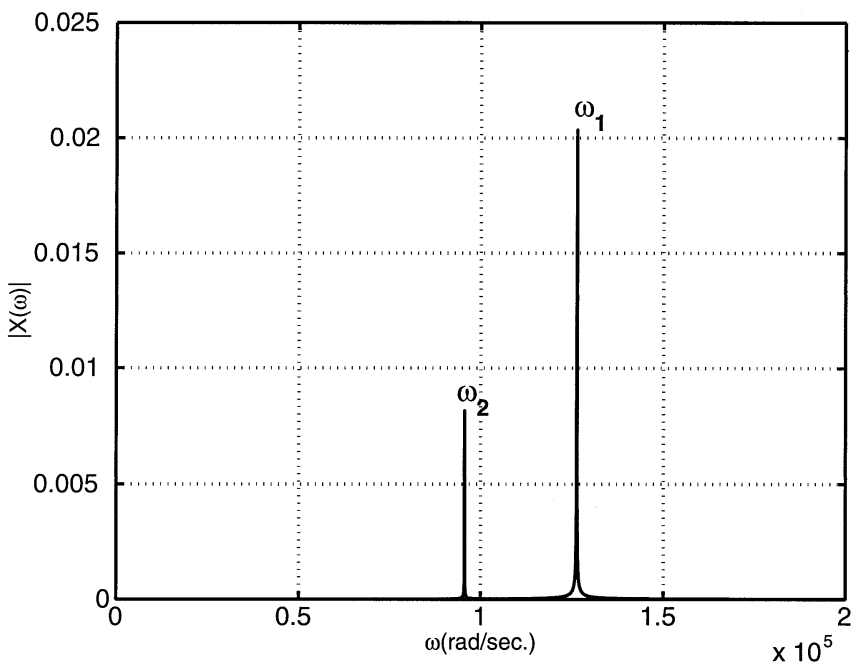

(b)

Fig. 8. (a) In the absence of damping, the trajectory of the gyroscope will form into elliptical type Lissajous figures. These figures will be bound by a rectangle oriented at an angle $\alpha$. PCA is used to determine the principal axes of elasticity, designated as $\bar{X}$ and $\bar{Y}$. (b) The Fourier transform of the $\mathrm{x}$ component of the data reveals two peaks due to off diagonal coupling of the stiffness matrix. From this plot, the $\mathrm{h}$ parameter of the system can be extracted as $2 h=m\left(\omega_{1}^{2}-\omega_{2}^{2}\right)$ where $\omega_{1}$ and $\omega_{2}$ correspond to the frequencies of the maximum and second maximum peaks, respectively.

We see that each position is comprised of the summation of two different sinusoidal functions due to the stiffness coupling. A plot of the time response of the system is a family of Lissajous figures [Fig. 8(a)]. Over time, it can be seen that the Lissajous figures will have trajectories bounded by a rectangle whose size is defined by $\mathrm{h}$ and which is oriented at the angle $\alpha$ from the coordinate axis. To determine the orientation of the principal axes, we employ the statistical method of principal component analysis (PCA).

Here, we will discuss the applications of PCA as it pertains to this study; a more general explanation can be found in [17]. In our case, we have two variables of interest, the $x$ position and y position. Now consider a $2 \times 1$ vector $\mathbf{V}=(x, y)$. We will assume that $\mathrm{x}$ and $\mathrm{y}$ have zero mean (centered about the origin) and that we have experimentally acquired covariances between $\mathrm{x}$ and $\mathrm{y}$. A covariance matrix $\mathbf{S}$ can be expressed by

$$
\mathbf{S}=\left[\begin{array}{l}
s_{x}^{2} s_{x y} \\
s_{x y} s_{y}^{2}
\end{array}\right]
$$

where $s_{x}^{2}$ and $s_{y}^{2}$ are the variances of $\mathrm{x}$ and $\mathrm{y}$ and the covariance $s_{x y}$ between $\mathrm{x}$ and $\mathrm{y}$ is given by

$$
s_{x y}=\frac{n \sum_{k=1}^{n} x_{k} y_{k}-\sum_{k=1}^{n} x_{k} \sum_{k=1}^{n} y_{k}}{[n(n-1)]}
$$

with the index of summation $\mathrm{k}$ going over the entire sample size $\mathrm{n}$. The covariance matrix is a numerical measure of the coupling between variables and in the case when $\mathbf{S}$ is diagonal, the vectors of $\mathbf{V}$ are uncorrelated, i.e., the $\mathbf{x}$ position has no influence on the $y$ position. Notice, when there is coupling through the stiffness matrix between the $\mathrm{x}$ and $\mathrm{y}$ position, the covariance matrix will also have coupling. Thus, a transformation that diagonalizes the covariance matrix will also diagonalize the stiffness matrix. We now introduce a coordinate transformation $\zeta=\mathbf{U}^{\mathbf{T}} \mathbf{V}$ where $\mathbf{U}$ is a constant matrix of transformation. It can be shown that there exists such an orthogonal transformation $\mathbf{U}$ that the covariance matrix $\tilde{\mathbf{S}}$ of this new coordinate system is [17]

$$
\tilde{\mathbf{S}}=E\left(\zeta \zeta^{\mathbf{T}}\right)=\mathbf{U}^{\mathbf{T}} \mathbf{S U}
$$

We will assume that the transformation $\mathbf{U}$ is a unity gain rotation and so $\mathbf{U}^{\mathbf{T}}=\mathbf{U}^{\mathbf{- 1}}$. With this assumption, we see that by choosing the columns of $\mathbf{U}$ to be the eigenvectors $\left(\mathbf{e}_{\mathbf{1}}\right.$ and $\left.\mathbf{e}_{2}\right)$ of the covariance matrix, we will achieve a diagonal form, thus the eigenvectors designate the basis vectors for the uncoupled space. Since $\mathbf{S}$ is Grammian, we are guaranteed of that eigenvectors are orthogonal. From (15), we can see that a rotation transformation of the form

$$
\left\{\begin{array}{l}
x \\
y
\end{array}\right\}=\left[\begin{array}{cc}
\cos \alpha & -\sin \alpha \\
\sin \alpha & \cos \alpha
\end{array}\right]\left\{\begin{array}{l}
q_{1} \\
q_{2}
\end{array}\right\}
$$

will uncouple the dynamic system to the uncorrelated principal axis coordinate system. It is necessary that this transformation must be equivalent to $\mathbf{U}^{\mathbf{T}}$ and so one expression for the eigenvectors representing the principal axes is [Fig. 8(a)]

$$
\begin{aligned}
& \mathbf{e}_{\mathbf{1}}=(\cos \alpha) \hat{e_{x}}-(\sin \alpha) \hat{e_{y}} \\
& \mathbf{e}_{2}=(\sin \alpha) \hat{e_{x}}+(\cos \alpha) \hat{e_{y}} .
\end{aligned}
$$

We can then directly calculate the angle $\alpha$ from the second eigenvector

$$
\alpha=\tan ^{-1}\left(\frac{e_{2, x}}{e_{2, y}}\right)
$$

where $e_{2, x}$ and $e_{2, y}$ are the $\mathrm{x}$ and $\mathrm{y}$ components of the second eigenvector, respectively.

Based off the dynamic system in (15), if we restrict the initial conditions to only an $x$ deflection $\left(y_{0}=0\right)$, then we are guaranteed of the system oscillating within a rectangle oriented in 
the boundary $\pi / 4>\alpha>-\pi / 4$. Taking a Fourier transform of (15) while considering only the $\mathrm{x}$ position yields

$$
\begin{aligned}
X(\omega) & =\frac{1}{2}\left(G(\alpha)+x_{0}\right) \pi \delta\left(\omega-\omega_{1}\right) \\
& +\frac{1}{2}\left(G(\alpha)-x_{0}\right) \pi \delta\left(\omega-\omega_{2}\right) \\
\omega_{1}^{2} & =\omega_{n}^{2}+\frac{h}{m} \\
\omega_{2}^{2} & =\omega_{n}^{2}-\frac{h}{m} .
\end{aligned}
$$

From (22), assuming the initial y deflection is zero, the ratio of the amplitudes of the two frequency components are

$$
\left|\frac{X\left(\omega_{1}\right)}{X\left(\omega_{2}\right)}\right|=x_{0} \frac{\cos (2 \alpha)+1}{\cos (2 \alpha)-1} .
$$

In this case, the highest peak in the frequency plot corresponds to the $\omega_{1}$ term and the second highest peak corresponds to the $\omega_{2}$ term [Fig. 8(b)]. Parameter $h$ and the ideal isotropic stiffness $k_{n}$ are calculated as $2 h=m\left(\omega_{1}^{2}-\omega_{2}^{2}\right)$ and $2 k_{n}=m\left(\omega_{1}^{2}+\omega_{2}^{2}\right)$, respectively. If $\omega_{1}<\omega_{2}$, then $h<0$.

At this point, the algorithm for determining $h$ and $k_{n}$ is dependent on differentiating the two peaks in the $\mathrm{X}$ frequency domain. As the errors, and hence the peak separation, tend to grow smaller, it becomes impossible to distinguish the two peaks and any numerical peak finding algorithm may result in erroneous results. A more accurate method for determining $\mathrm{h}$ and $k_{n}$ requires the calculated $\alpha$ from the PCA analysis. Using the transformation from (19), the equations in the transformed principal coordinate space are

$$
\begin{aligned}
& q_{1}(t)=\left(2 x_{0} \cos \alpha+2 y_{0} \sin \alpha\right) \cos \omega_{q 1} t \\
& q_{2}(t)=\left(-2 x_{0} \sin \alpha+2 y_{0} \cos \alpha\right) \cos \omega_{q 2} t .
\end{aligned}
$$

Thus, if we transform our data in this way and then take the Fourier transform, we see that there will be one peak for $q_{1}$ and $q_{2}$ at frequencies $\omega_{q 1}$ and $\omega_{q 2}$, respectively. These frequencies are

$$
\begin{aligned}
& \omega_{q 1}^{2}=\omega_{n}^{2}+\frac{h}{m} \\
& \omega_{q 2}^{2}=\omega_{n}^{2}-\frac{h}{m} .
\end{aligned}
$$

Then, $\mathrm{h}$ and $k_{n}$ are calculated simply as $2 h=m\left(\omega_{q 1}^{2}-\omega_{q 2}^{2}\right)$ and $2 k_{n}=m\left(\omega_{q 1}^{2}+\omega_{q 2}^{2}\right)$, respectively. This result makes it easier to identify smaller errors since it is only necessary to identify the largest peak in each frequency spectrum rather than the largest two.

The benefits of this algorithm is since the PCA and Fourier transforms take advantage of all the data, it is not necessary to have precise deflection information. This is especially advantageous in systems where small deflections are difficult to resolve due to noise in the sensing electronics. One of the shortcomings of this algorithm is that systems with high damping reduce the amount of data points, resulting in erroneous results. A solution to highly damped systems is vacuum packaging of the device and also to employ an energy compensating controller.
Ambiguity of the Algorithm: In our derivation of the angle $\alpha$ from (20), we initially neglected the fact that the eigenvectors are interchangeable in the transformation matrix. Thus, another possible rotation angle is

$$
\alpha_{2}=\cot ^{-1}\left(\frac{-e_{1, x}}{e_{1, y}}\right)
$$

where $\alpha_{2}$ is the angle calculated if we were to use the first eigenvector rather than the second. Here, $\alpha_{2}$ is related to the $\alpha$ calculated from (21) by $\alpha_{2}=\alpha-\operatorname{sign}\{\alpha\}(\pi / 2)$. Since the output from the PCA analysis is numerical values for the eigenvectors, it is impossible to tell if the output PCA angle is either $\alpha$ or $\alpha-\operatorname{sign}\{\alpha\}(\pi / 2)$. However, we will show that even with this ambiguity in the angle, we will still arrive at an equivalent solution.

From (23), if we make the substitution $\alpha=\alpha-$ $\operatorname{sign}\{\alpha\}(\pi / 2)$, then the equation becomes

$$
\left|\frac{X\left(\omega_{1}\right)}{X\left(\omega_{2}\right)}\right|=x_{0} \frac{\cos (2 \alpha)-1}{\cos (2 \alpha)+1} .
$$

We see that the conditions are changed and now $\omega_{2}$ corresponds to the first maximum peak and $\omega_{1}$ corresponds to the second maximum peak. Now the algorithm will calculate $-h$ rather than $h$. If we substitute the two angle and $\mathrm{h}$ combinations of $(\alpha, h)$ and $(\alpha-\operatorname{sign}\{\alpha\}(\pi / 2),-h)$ into (11), we will arrive at equivalent solutions. Thus, as long as the guess for $\alpha$ is between $-\pi / 4$ and $\pi / 4$, the algorithm will correctly identify the structural nonidealities whether the initial guess for $\alpha$ was correct or if it was actually $\alpha-\operatorname{sign}\{\alpha\}(\pi / 2)$.

\section{B. Energy Compensating Controller}

In reality, viscous damping will eventually deplete the energy of the system, resulting in a finite number of cycles from which to obtain data. In relatively high quality factor systems $(\mathrm{Q}>$ 100), there is sufficient energy to obtain enough data before the oscillation pattern dies out. However, in cases of low quality systems $(Q<100)$, there are not enough cycles to obtain an accurate calculation of the anisoelasticities. For example, such Q values are indicative of operating microstructures in an ambient atmosphere [18]. In these cases, a controller to compensate for energy losses is required. In the angular gyroscope, a controller of the form [10]

$$
\left\{\begin{array}{l}
F_{x} \\
F_{y}
\end{array}\right\}=-\gamma_{2} \cdot \Delta E \cdot\left\{\begin{array}{l}
\dot{x} \\
\dot{y}
\end{array}\right\}
$$

is implemented into (9) that guarantees to maintain the energy of the system without interfering with the identification of anisoelasticities. Here, $\gamma_{2}$ is a constant gain, $\dot{x}$ and $\dot{y}$ are the velocities along the $\mathrm{X}$ and $\mathrm{Y}$ directions, and $\Delta E$ is the change in system energy given as

$$
\Delta E=E_{0}-\frac{\omega_{n}^{2}\left(x^{2}+y^{2}\right)+\left(\dot{x}^{2}+\dot{y}^{2}\right)}{2}
$$

where $E_{0}$ denotes nominal energy of the system normalized with respect to the effective mass. In using the controller, we have assumed isotropic damping with no coupling. This controller is based on small parameter variations [14] and in order 


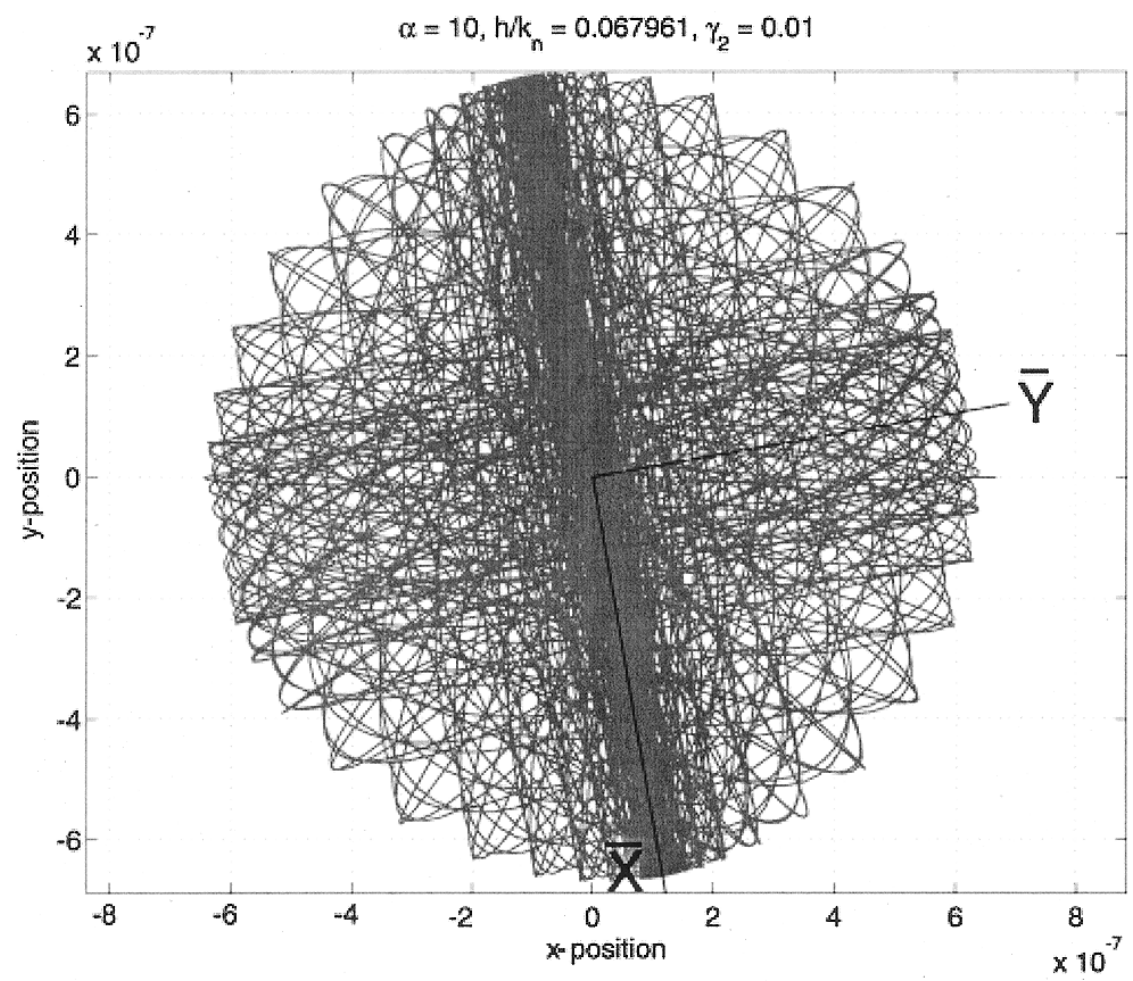

(a)

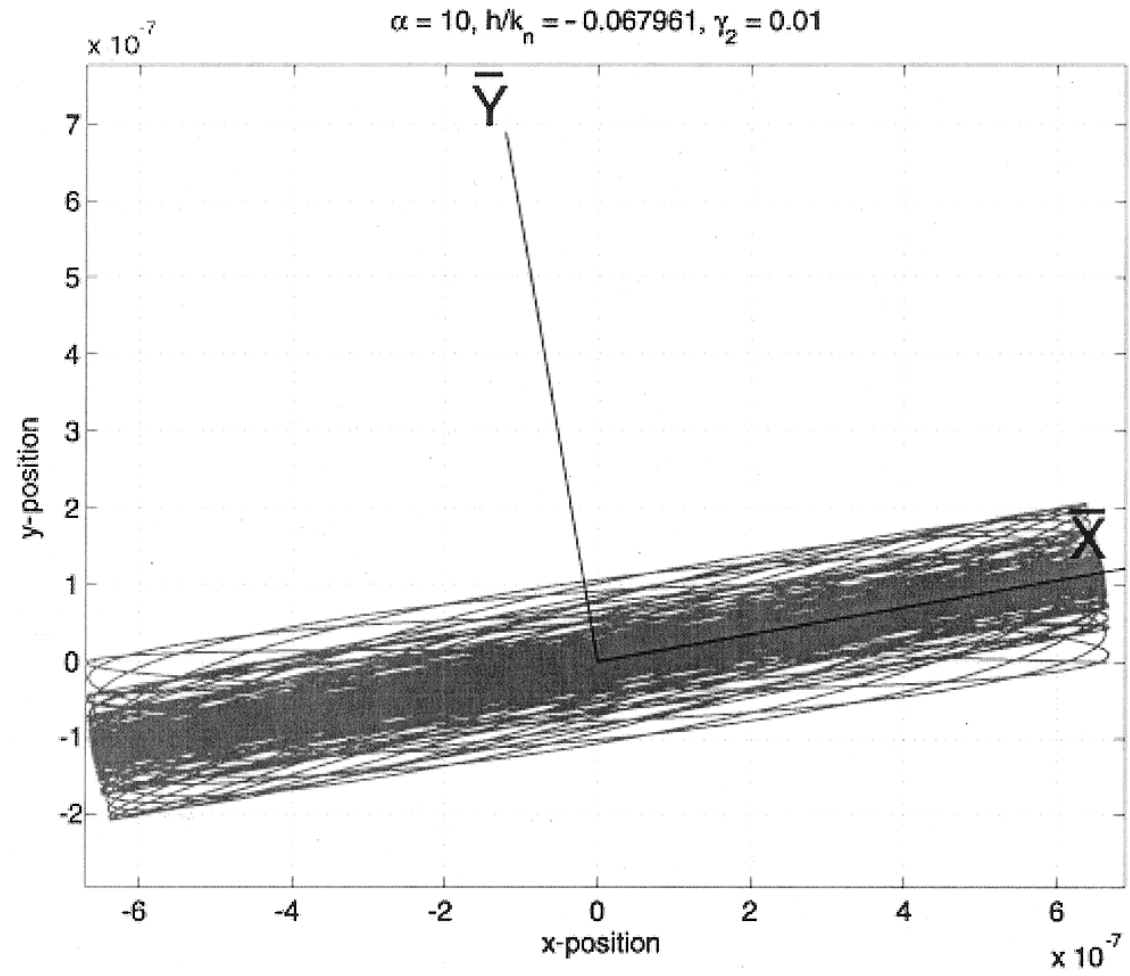

(b)

Fig. 9. With the implementation of an energy compensating controller, the systems tends toward the principal axis with the lowest stiffness. In both of these cases, $\alpha$ is the same, but the mismatch in the principal axis stiffness values is different: (a) $h>0$ and (b) $h<0$.

for the controller not to interfere with the measured parameters, the gain $\gamma_{2}$ must be sufficiently small.

With the implementation of this controller, the oscillation of the system tends to propagate toward the principal axis with the smallest stiffness (Fig. 9). We will assume that this is the $\bar{x}$ axis (see Fig. 4), which is misaligned from the $\mathrm{x}$-axis by the angle $\alpha$ and is guaranteed of being oriented in the range of $-\pi / 2<\alpha<$ $\pi / 2$. Now, we consider the dynamics in the transformed principal axis coordinate system from (24). Taking the Fourier transform of $q_{1}$ and $q_{2}$ yields one peak for each at frequencies $\omega_{q 1}$ and 


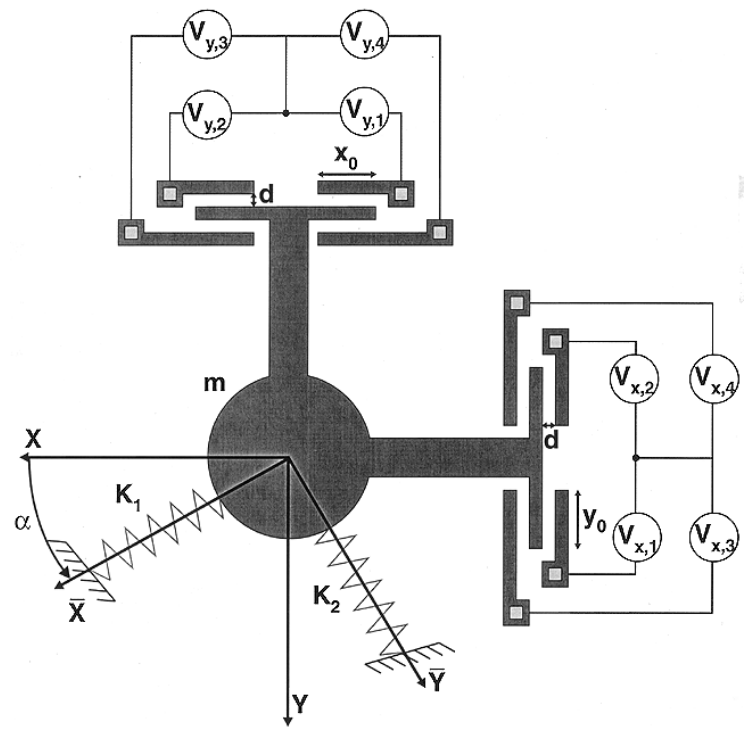

Fig. 10. We use the inherent nonlinearity of the parallel plate actuators along the $x$ and $y$ directions in order to tune out the nonideal stiffness elements.

$\omega_{q 2}$, respectively. From (25), $\mathrm{h}$ is given as $2 h=m\left(\omega_{q 1}^{2}-\omega_{q 2}^{2}\right)$ and $k_{n}$ is calculated by $2 k_{n}=m\left(\omega_{q 1}^{2}+\omega_{q 2}^{2}\right)$. The difference between this and the algorithm of the previous section is that we are assuming that $q_{1}$ is always the axis with the smallest stiffness. Thus, $\omega_{q 1}^{2}$ is always less than $\omega_{q 2}^{2}$, so $h$ is limited to being always less than zero. However, with the new limits of $\alpha$ from $-\pi / 2$ and $\pi / 2$, we have compensated for this restriction on $h$ and the algorithms will still correctly calculate the stiffness nonidealities when substituting into (11).

The advantages of this methodology are the same as the previous algorithm in Section IV-A with the addition of energy compensation. As a result, systems with low Q can still be analyzed to determine the structural anisoelasticities. In addition, no initial deflection is required and the controller will tend to drive the system to a predetermined energy level. The caveat to this method is that the chosen gain of the energy compensation controller must be sufficiently small to avoid interfering with the measurement of the anisoelasticity.

\section{Dual Stage Electrostatic Control}

Having obtained the anisoelasticity parameters from the algorithm of the previous section, we now demonstrate how this is applied to a feedforward type architecture using electrostatic nonlinear parallel plates.

\section{A. Electrostatic "Trimming"}

Two different physical mechanisms have been reported in MEMS for active frequency tuning: thermal compensation [19] and electrostatic tuning [2]. Since the angular gyroscope utilizes parallel plate electrodes for drive and sense, we focus on using the inherent nonlinearity of the electrostatic forces to tune out the nonideal components of the stiffness matrix (Fig. 10). The nonideal dynamics of the gyroscope, including the electrostatics, can be expressed by

$$
\begin{aligned}
& m \ddot{x}+\left(k_{n}+h \cos 2 \alpha\right) x+(h \sin 2 \alpha) y=F_{e, x} \\
& m \ddot{y}+(h \sin 2 \alpha) x+\left(k_{n}-h \cos 2 \alpha\right) y=F_{e, y}
\end{aligned}
$$

where $F_{e, x}$ and $F_{e, y}$ are electrostatic forces expressed as

$$
\begin{aligned}
\frac{2 F_{e, i}}{\epsilon_{0} t}= & \frac{g-N j}{(d-i)^{2}} V_{i, 1}^{2}+\frac{g+N j}{(d-i)^{2}} V_{i, 2}^{2} \\
& -\frac{g-N j}{(d+i)^{2}} V_{i, 3}^{2}-\frac{g+N j}{(d+i)^{2}} V_{i, 4}^{2} \\
& +\frac{N}{d-j} V_{j, 1}^{2}-\frac{N}{d-j} V_{j, 2}^{2} \\
& -\frac{N}{d+j} V_{j, 3}^{2}+\frac{N}{d+j} V_{j, 4}^{2} .
\end{aligned}
$$

Here, $\mathrm{i}=\{\mathrm{x}, \mathrm{y}\}, \mathrm{j}=\{\mathrm{y}, \mathrm{x}\}, \mathrm{N}$ is the number of control electrode sets (e.g., in Fig. 10, $\mathrm{N}=1$ ), $t$ is the structural thickness, $d$ is the parallel plate gap, $g$ is the total plate overlap ( $\left.g=N x_{0}=N y_{0}\right)$, and $\epsilon_{0}$ is the permittivity of a vacuum. For structural compensation, we use the following control voltages:

$$
\begin{aligned}
& V_{x, 1}=V_{\mathrm{DC}}+v_{x x}+v_{q}+v_{x} \quad V_{x, 2}=V_{\mathrm{DC}}+v_{x x}-v_{q}+v_{x} \\
& V_{x, 3}=V_{\mathrm{DC}}+v_{x x}-v_{q}-v_{x} \quad V_{x, 4}=V_{\mathrm{DC}}+v_{x x}+v_{q}-v_{x} \\
& V_{y, 1}=V_{\mathrm{DC}}+v_{y y}-v_{q}+v_{y} V_{y, 2}=V_{\mathrm{DC}}+v_{y y}+v_{q}+v_{y} \\
& V_{y, 3}=V_{\mathrm{DC}}+v_{y y}-v_{q}-v_{y} V_{y, 4}=V_{\mathrm{DC}}+v_{y y}+v_{q}-v_{y}
\end{aligned}
$$

where $V_{\mathrm{DC}}$ is a constant bias voltage, $v_{q}$ is a constant feedforward misalignment control voltage, $v_{x x}$ and $v_{y y}$ are constant stiffness mismatch control voltages, and $v_{x}$ and $v_{y}$ are state dependent feedback control voltages. We determine the voltages for the feedforward control by first assuming zero feedback $\left(v_{x}=v_{y}=0\right)$. The nonlinearity of the parallel plates leads to a nonzero first derivative of the electrostatic force with respect to position, which can be interpreted as an electrostatic spring [2], contributing to the overall system stiffness. Additionally, the net forces and moments are zero, so the stiffness can be tuned without deflecting the structure. In order to find a closed-form approximation for the control voltages, we assume small deflections and combine the ideal $\left(K_{i}\right)$, nonideal $\left(K_{n}\right)$, and electrostatic $\left(K_{e}\right)$ matrix contributions together to form the overall stiffness realization

$$
\begin{aligned}
K & =K_{i}+K_{n}+K_{e} \\
K_{i} & =\left[\begin{array}{cc}
k_{n} & 0 \\
0 & k_{n}
\end{array}\right] \\
K_{n} & =\left[\begin{array}{cc}
h \cos (2 \alpha) & h \sin (2 \alpha) \\
h \sin (2 \alpha) & -h \cos (2 \alpha)
\end{array}\right] \\
K_{e} & =\frac{4 \epsilon_{0} t}{d^{2}}\left[\begin{array}{ll}
\Phi_{1} & \Phi_{2} \\
\Phi_{2} & \Phi_{3}
\end{array}\right] \\
\Phi_{1} & =-\frac{g}{d}\left(\left(V_{\mathrm{DC}}+v_{x x}\right)^{2}+v_{q}^{2}\right) \\
\Phi_{2} & =v_{q}\left(\left(V_{\mathrm{DC}}+v_{x x}\right)+\left(V_{\mathrm{DC}}+v_{y y}\right)\right) \\
\Phi_{3} & =-\frac{g}{d}\left(\left(V_{\mathrm{DC}}+v_{y y}\right)^{2}+v_{q}^{2}\right) .
\end{aligned}
$$

With any arbitrary dc voltage, there exist different sets of control voltages $v_{q}, v_{x x}$, and $v_{y y}$ to cancel the off-diagonal terms of the stiffness matrix and set the on-diagonal stiffness terms equal to each other $\left(k_{\text {tuned }}\right)$. Setting the off-diagonal terms equal to zero leads to the following constraint on the the misalignment control voltage $v_{q}$ :

$$
v_{q}=\frac{-1}{4} \frac{h d^{2} \sin 2 \alpha}{\epsilon_{0} t N\left(\left(V_{\mathrm{DC}}+v_{x x}\right)+\left(V_{\mathrm{DC}}+v_{y y}\right)\right)} .
$$




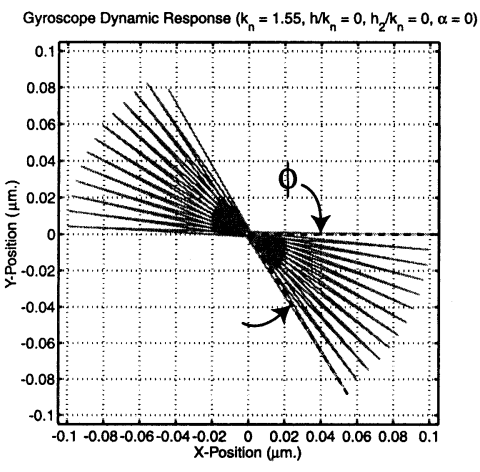

(a)

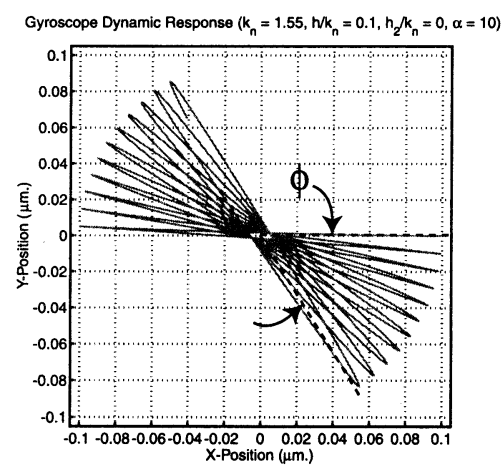

(d)

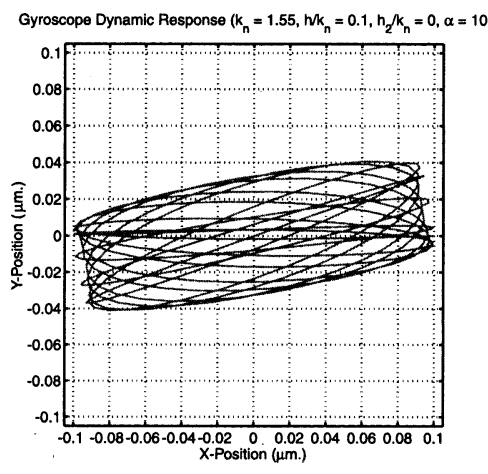

(b)

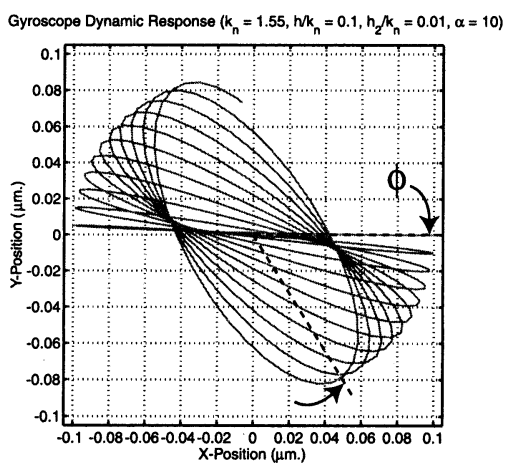

(e)

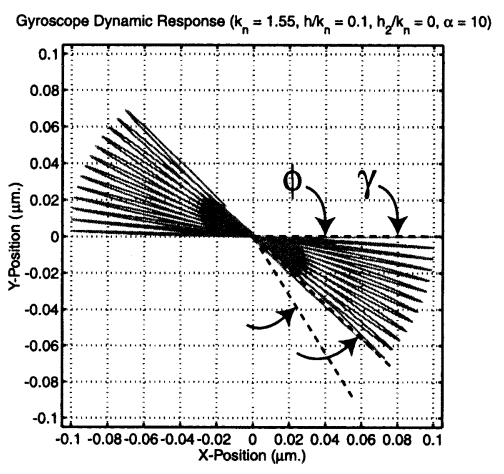

(c)

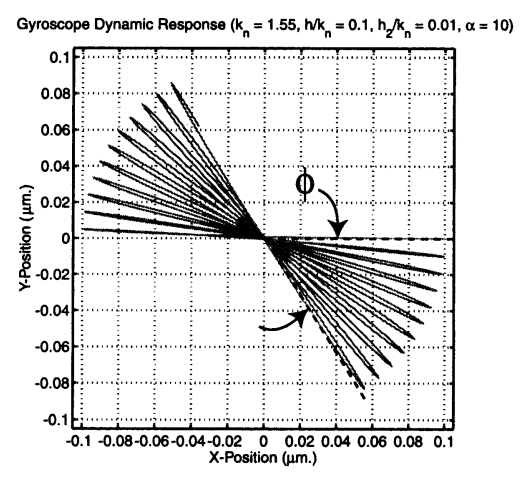

(f)

Fig. 11. (a) In the absence of imperfections, the line of oscillation precesses normally by angle $\phi$ in the presence of an input angular rotation. (b) Large anisoelasticities due to fabrication imperfections interfere with the ideal operation of the device, eliminating the precession. (c) The feedback control used to compensate for these large imperfections also interferes with the precession pattern, reducing the precession angle from the ideal angle $\phi$ to $\gamma$. (d) By using a feedforward control, large structural anisoelasticities can be eliminated while maintaining the ideal precession of the device. (e) While the feedforward control can compensate for constant structural imperfections, it is invariant to small perturbation that arise during normal operation of the device (e.g., due to thermal fluctuations), which disrupt the line of oscillation. (f) A dual-stage feedforward/feedback architecture can compensate for both structural imperfections and small perturbations without interfering with the precession.

There are several ways of choosing $v_{x x}$ and $v_{y y}$, which are constrained by the fact that the on-diagonal terms must be identical and that voltage values must be real. One way is to set the voltage which influences the smallest of the on-diagonal stiffness terms equal to zero and use the second voltage to tune the larger stiffness term to match. This gives the following set of control voltages for $h \cos 2 \alpha \geq 0$ :

$$
\begin{aligned}
& v_{x x}=-V_{\mathrm{DC}}+\left|\sqrt{V_{\mathrm{DC}}^{2}+\frac{h d^{3} \cos 2 \alpha}{2 \epsilon_{0} t g}}\right| \\
& v_{y y}=0
\end{aligned}
$$

and for $h \cos 2 \alpha \leq 0$

$$
\begin{aligned}
& v_{x x}=0 \\
& v_{y y}=-V_{\mathrm{DC}}+\left|\sqrt{V_{\mathrm{DC}}^{2}-\frac{h d^{3} \cos 2 \alpha}{2 \epsilon_{0} t g}}\right| .
\end{aligned}
$$

Since the on-diagonal electrostatic stiffness term is always negative, the tuned on-diagonal stiffness value $k_{\text {tuned }}$ will always be less than the original ideal stiffness $\left(k_{\text {tuned }}<k_{n}\right)$. To take this into account, a good strategy is to design the gyroscope suspension to be stiffer than desired. A theoretical limit is reached when these stiffness terms become negative $\left(k_{\text {tuned }}<0\right)$ and the system becomes unstable. Thus, an optimal dc bias voltage is one that maximizes the trace of the stiffness matrix subject to the constraint that $v_{q}, v_{x x}$, and $v_{y y}$ must satisfy (32) and (33)/(34). A closed form solution is not readily available for this voltage, and must be solved for numerically. Even with an optimal choice for voltages, invariably a practical limit is reached where errors cannot be compensated for without resulting in system instability. This occurs when the compensated on-diagonal terms are less than or equal to zero.

\section{B. Feedback Control}

While the feedforward control can compensate for large constant structural imperfections, it is also necessary to implement a feedback control to correct for small fluctuations that arise during the normal operation of the device. For the feedback control, we implement state dependent control voltages $v_{x}$ and $v_{y}$

$$
\begin{aligned}
& v_{x}=\frac{d^{2}\left(N d v_{q} F_{y}+F_{x} g\left(V_{\mathrm{DC}}+v_{x x}\right)\right)}{4 \epsilon_{0} t\left(g^{2}\left(V_{\mathrm{DC}}+v_{x x}\right)\left(V_{\mathrm{DC}}+v_{y y}\right)-N^{2} d^{2} v_{q}^{2}\right)} \\
& v_{y}=\frac{d^{2}\left(N d v_{q} F_{x}+F_{y} g\left(V_{\mathrm{DC}}+v_{y y}\right)\right)}{4 \epsilon_{0} t\left(g^{2}\left(V_{\mathrm{DC}}+v_{x x}\right)\left(V_{\mathrm{DC}}+v_{y y}\right)-N^{2} d^{2} v_{q}^{2}\right)}
\end{aligned}
$$

where $F_{x}$ and $F_{y}$ are the control forces based off the skew symmetric control architecture given in (13). This architecture is guaranteed of compensating for small perturbations without interfering with the Coriolis induced precession. With the addition of the feedforward control voltages, the complete dual stage architecture is capable of "trimming" large structural imperfections while compensating for small perturbations. Computer simulations are presented to demonstrate the effectiveness of this control strategy. 


\section{Numerical Simulation}

All simulation parameters are based off a realistic implementation of a surface micromachined rate integrating gyroscope (Fig. 7). The mass of the device is $4.10 \times 10^{-10} \mathrm{~kg}$ and the isotropic stiffness $k_{n}$ is $1.55 \mathrm{~N} / \mathrm{m}$, giving a natural frequency of $9.8 \mathrm{kHz}$ for the system. The total parallel plate overlap $\mathrm{g}$ is $1200 \mu \mathrm{m}$, the parallel plate gap $\mathrm{d}$ is $2 \mu \mathrm{m}$, and the permittivity $\epsilon_{0}$ is $8.854 \times 10^{-12} \mathrm{~F} / \mathrm{m}$. The simulations based off the nonlinear equations of motion (30) are run for $10 \mathrm{~ms}$, assuming an initial $x$ deflection of .1 microns to satisfy the small deflection assumption. For a chosen set of large structural anisoelasticities $\left(h / k_{n}=10 \%, \alpha=10^{\circ}\right)$, an optimal dc bias voltage which maximizes the trace of the stiffness matrix is calculated to be 4.20 $\mathrm{V}$, which will remain constant for each simulation presented here. Under this bias voltage and in the absence of imperfections ( $h=0, \alpha=0$ ), the line of oscillation precesses by an angle $\phi$ [Fig. 11(a)]. With large structural anisoelasticities $\left(h / k_{n}=\right.$ $\left.10 \%, \alpha=10^{\circ}\right)$ and without compensation $\left(v_{q}=v_{x x}=v_{y y}=\right.$ $v_{x}=v_{y}=0$ ), the system oscillates about the principal axes of elasticity and there is no precession [Fig. 11(b)]. A purely feedback control using control voltages $v_{x}$ and $v_{y}$ as calculated from (35) is then attempted, which eliminates the quadrature error, but also interferes with the precession angle [Fig. 11(c)]. Next, appropriate compensating feedforward control voltages $v_{q}, v_{x x}$, and $v_{y y}$ are chosen based off the assumption that $\alpha$ and $\mathrm{h}$ are well known. The purely feedforward control $\left(v_{x}=v_{y}=0\right)$ is used to eliminate the quadrature error, which restores ideal precession of the line of oscillation [Fig. 11(d)]. However, based on simulation of the PCA and Fourier anisoelasticity finding algorithms from Section III, the anisoelasticity parameters are actually calculated to be $\left(h / k_{n}\right)_{\text {calc }}=10.23 \%, \alpha_{\text {calc }}=10.16^{\circ}$. To realize the effects of this error, as well as other small perturbations that could arise during normal operation, we add small anisoelasticities $\left(h_{2} / k_{n}=1 \%\right)$ to the system that cannot be compensated for by the feedforward control. This results in destruction of the precession pattern [Fig. 11(e)]. The state dependent voltages are then included to compensate for these perturbations while still allowing the undisturbed precession pattern [Fig. 11(f), note that the precession angle is the same as Fig. 11(a) and Fig. 11(f)].

\section{CONCLUSION}

In this paper, we have demonstrated the necessity for a dual stage control architecture comprising feedforward and feedback control systems in order to compensate for fabrication imperfections and in-operation perturbations prevalent in micromachined gyroscopes. We have shown how this control can be realized in a gyroscope using electrostatic parallel plate actuators. The successful application of feedforward control was shown in a device with large structural imperfections of $10 \%$ of the ideal stiffness where a purely feedback control would interfere with the performance of the device. Additionally, the feedback portion of the control was shown to compensate for a further $1 \%$ error to the stiffness as a result of small perturbations arising during normal operation. This type of compensation is limited by the stability of the device, as large voltages will result in an unstable system. In the case of devices with imperfections beyond this limit, it is necessary to employ alternative methods such as thermal tuning or postprocessing. Future work includes study of additional capabilities for identification and suppression of damping and experimental demonstration of the dual stage architecture using a DSP implementation.

\section{REFERENCES}

[1] N. Yazdi, F. Ayazi, and K. Najafi, "Micromachined inertial sensors," Proc. IEEE, vol. 86, pp. 1640-1659, Aug. 1998.

[2] W. A. Clark, "Micromachined Vibratory Rate Gyroscopes," Ph.D dissertation, Univ. California, Berkeley, 1997.

[3] M. Putty and K. Najafi, "A micromachined vibrating ring gyroscope," in Proc. IEEE Solid State Sens. Actuators Workshop, Hilton Head Island, SC, June 1994, pp. 213-220.

[4] S. Park and R. Horowitz, "Adaptive control for the conventional mode of operation of MEMS gyroscopes," J. Microelectromech. Syst., vol. 12, no. 1, pp. 101-108, Feb. 2003

[5] V. P. Veiko, "Laser-assisted microshaping," in Proc. SPIE: Laser-Assisted Microtechnol., vol. 4157, St. Petersburg-Pushkin, Russia, Aug. 2000, pp. 93-104.

[6] T. Lam and R. B. Darling, "Modeling of focused ion beam trimming of cantilever beams," in Proc. 3rd Int. Conf. Modeling and Simulation of Microsystems, San Diego, CA, Mar. 2000.

[7] D. Joachim and L. Lin, "Selective polysilicon deposition for frequency tuning of MEMS resonators," in IEEE Microelectromech. Syst., Las Vegas, NV, Jan. 2002

[8] A. Shkel and R. T. Howe, "Polysilicon Surface Micromachined Rate Integrating Gyroscopes," U.S. Patent 6481 285, Nov. 2002.

[9] A. Shkel, "Smart MEMS: Micro-structures with error-suppression and self-calibration control capabilities," in Amer. Control Conf., Arlington, CA, June 2001.

[10] A. Shkel, R. Horowitz, A. Seshia, and R. T. Howe, "Dynamics and control of micromachined gyroscopes," in Proc. Amer. Control Conf., San Diego, CA, June 1999, pp. 2119-2124.

[11] B. Friedland and M. Hutton, "Theory and error analysis of vibrating-member gyroscope," IEEE Trans. Automat. Contr., vol. AC-23, pp. 545-556, Apr. 1978.

[12] A. Shkel, R. T. Howe, and R. Horowitz, "Modeling and simulation of micromachined gyroscopes in the presence of imperfections," in Int. Conf. Modeling and Simulation of Microsystems, San Juan, PR, Apr. 1999, pp. 605-608.

[13] I. V. Novozhilov, Fractional Analysis: Methods of Motion Decomposition. Boston, MA: Birkhäuser, 1997.

[14] V. F. Zhuravlev, "Oscillation shape control in resonant systems," J. Appl. Math. Mech., vol. 56, no. 5, pp. 725-735, 1992.

[15] - "Theoretical foundations of solid-state wave gyroscopes," Mech. Solids, vol. 28, no. 3, pp. 3-15, 1993.

[16] R. T. M'Closkey, S. Gibson, and J. Hui, "System identification of a MEMS gyroscope," J. Dynamic Syst., Meas., Contr., vol. 123, pp. 201-210, June 2001.

[17] A. Basilevsky, Statistical Factor Analysis for Related Methods, Theory and Applications. New York: Wiley, 1994.

[18] Y.-H. Cho, B. M. Kwak, A. P. Pisano, and R. T. Howe, "Viscous energy dissipation in laterally oscillating planar microstructures: A theoretical and experimental study," IEEE Proc. Microelectromech. Syst. Workshop, Feb. 1993.

[19] T. Remtema and L. Lin, "Active frequency tuning for microresonators by localized thermal stressing effects," in Solid-State Sens. Actuator Workshop, Hilton Head Island, SC, June 2000, pp. 363-366.

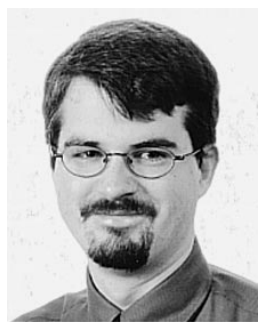

Chris C. Painter (S'02) received the B.S. degree in mechanical engineering from the University of California, San Diego, in 1999 and the M.S. degree in mechanical and aerospace engineering from the University of California, Irvine (UCI), in 2000. He is currently pursuing the Ph.D. degree.

After receiving the M.S. degree, he joined the Microsystems Laboratory, UCI, developing a MEMS-based absolute angle measuring gyroscope as the focus of his Ph.D. work. He is the author of several patents (pending) in the field and has over 10 publications in various international conferences and journals. $\mathrm{He}$ is the recipient of a National Defense Science and Engineering Graduate Fellowship sponsored by the Department of Defense and has served as a reviewer for several major journals. 


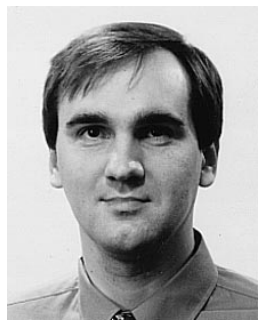

Andrei M. Shkel (A'98) received the Diploma (with excellence) in mechanics and mathematics from Lomonosov's Moscow State University, Moscow, Russia, in 1991 and the Ph.D. degree in mechanical engineering from the University of Wisconsin, Madison, in 1997.

He is on the faculty at the University of California, Irvine (UCI), where he is an Assistant Professor in the Department of Mechanical Aerospace Engineering, in the Department of Electrical Engineering Computer Sciences, and in the Department of Biomedical Engineering. He is also the Director of the UCI Micro-Systems Laboratory. After receiving the Ph.D. degree, he joined the Berkeley Sensor Actuator Center (BSAC) as a Postdoctoral Researcher. He then held research and consulting positions in several high-tech and venture companies, including MEMSolutions, Inc., Solus Microtechnologies, VIP Sensors, Silicon Valley Venture, etc. His professional interests, reflected in over 50 publications, include solid-state sensors and actuators, MEMS-based neuroprosthetics, sensor-based intelligence, and control theory. He currently holds three U.S. patents on micromachined angle-measuring gyroscope, design and fabrication of monolithic optical switches, and hybrid surface micromachining processes. $\mathrm{He}$ is a member of the Editorial Advisory Board (EAB) for the ISA magazine - SensorTech,

Dr. Shkel is an associate member of the ASME and SPIE. He has served on a number of editorial boards, including Guest Editor for two special issues of the IEEE SENSORS JouRnAL. He was Publications Chair of the 2002 IEEE Sensors Conference, Vice General Chair and Publication Chair of the 2003 IEEE Sensors Conference, and a member of technical committees of 2001, 2002, and 2003 SPIE, TMS 2003, and ACC 2001. He was awarded the 2002 George E. Brown, Jr. Award and was the recipient of 2001 Fellowship of the Japanese Advanced Science Institute. 